\title{
Cell apoptosis specific marker found by Fourier Transform Infrared Spectroscopy
}

\author{
Silvia Gaudenzi ${ }^{\text {a }}$, Deleana Pozzi ${ }^{\mathrm{b}}$, Paolo Toro ${ }^{\mathrm{a}}$, Ida Silvestri ${ }^{\mathrm{b}}$, Stefania Morrone $^{\mathrm{b}}$ \\ and Agostina Congiu Castellano ${ }^{\mathrm{c}, *}$ \\ a Dipartimento di Fisica, Università di Roma "La Sapienza", Roma, Italy \\ ${ }^{\mathrm{b}}$ Dipartimento di Medicina Sperimentale e Patologia, Università di Roma "La Sapienza", Roma, Italy \\ ' INFM and Dipartimento di Fisica, Università di Roma "La Sapienza", Roma, Italy
}

\begin{abstract}
We used Fourier Transform Infrared Spectroscopy (FTIR) combined with flow cytometry to study the apoptosis and necrosis processes in Jurkat, a lymphocyte cell line. The apoptosis was induced in the cells by a chemical agent, the actinomycin $\mathrm{D}$, while the necrosis was induced lowering the $\mathrm{pH}$ value to 4.2. The apoptotic events were analysed by flow cytometry (using annexin $\mathrm{V}$ and propidium iodide) and contemporary monitored by FTIR spectroscopy at different times after the treatment. This comparison allowed us to find in the IR spectrum, between $3000 \mathrm{~cm}^{-1}$ and $2800 \mathrm{~cm}^{-1}$, a "marker band" of the apoptosis corresponding to the exposure of phosphatidylserine on the outer leaflet of the membrane. A marker of a specific cellular process obtained by using a non-destructive technique such as FTIR spectroscopy, has a great significance in the diagnostic medicine providing a tool for detecting pathologies in vivo.
\end{abstract}

Keywords: FTIR spectroscopy, apoptosis, necrosis, cell membrane

\section{Introduction}

Providing a "molecular fingerprint" infrared spectroscopy is considered a diagnostic and monitoring tool in biology and medicine. The observed spectroscopic changes are related to changes in the concentration and conformational orientation of functional groups associated with proteins, lipids, nucleic acids and carbohydrates [7]. The most important feature of infrared spectroscopy is that it represents a non-destructive technique allowing to obtain simultaneous information on all macromolecules in a cell population within few seconds.

Therefore this technique is a sensitive tool to study cellular processes such as apoptosis, cell differentiation $[3,6,11]$ and conformational changes induced by various agents.

We have shown recently [2] that the toxic effect induced in Jurkat cells treated with cadmium compounds, is revealed both by FTIR and by a classical biological method. Similarly in this paper we study the apoptosis and necrosis in Jurkat cells by using at the same time a biological assay such as the flow cytometry and a physical technique such as FT-IR spectroscopy.

Apoptosis is a physiological cell death mechanism whose failure causes many pathological conditions such as anomalous cells proliferation, degeneration or inflammation $[4,5,12]$.

\footnotetext{
*Corresponding author: A. Congiu Castellano, Dipartimento di Fisica, Università di Roma "La Sapienza", Piazzale A. Moro 2, 00185 Roma, Italy. Tel.: +39 06 49913503; Fax: +39 06 4463158; E-mail: a.congiu@ @aspur.it.
} 
Apoptosis can be induced in cell culture by the exposure to different agents (chemical compounds, UV radiation, deprivation of essential growth factors, etc.); we report in this paper a study concerning the apoptosis process induced in Jurkat cells by actinomycin D treatment.

In apoptotic cells many changes in membrane fluidity, ionic charge, membrane proteins conformation and lipid asymmetry are detected; thus the cells undergoing apoptosis show a morphology with some specific markers. A well-studied marker of apoptosis is the exposure to the outer leaflet of the plasma membrane of phosphatidylserine [1], an anionic phospholipid usually confined to the interior of the cell.

The combination of two experimental methods (FTIR and flow cytometry) has been necessary to reach our primary goal that was to find in the IR spectrum "a marker band" monitoring the apoptotic and necrotic process. The existence of a specific marker revealed by a non-invasive technique has a great significance in the diagnostic medicine providing a tool for detecting pathologies in vivo.

Different features in IR spectra of apoptotic and necrotic cells are expected, according to different pathways of the cellular death due to the two processes. In fact during apoptosis the cells undergo nuclear and cytoplasmatic shrinkage, the chromatin is partitioned into multiple fragments, DNA and RNA are segregated and packed into different granules which form apoptotic bodies, while the cell membrane maintains its integrity. On the contrary, the rapid loss of the membrane integrity and cell swelling characterize the necrotic cell's death.

Our preliminary effort has been devoted to point out the protocols of the experimental procedures to obtain IR spectra reproducibility. In particular the reproducibility depends from different parameters: an important variable is the different growth stage of the cells [6]. Therefore it has been necessary to plan adequately the experiments in order to attribute the spectral differences only to apoptosis or necrosis processes, not to different stages of the cell cycle.

\section{Materials and methods}

\subsection{Cell culture and treatments}

Lymphoid T-cell, Jurkat, CD3 ${ }^{+} / \mathrm{CD} 2^{+}[10]$ were grown in RPMI 1640 supplemented with $10 \%$ fetal bovine serum and penicillin-streptomycin at $37^{\circ} \mathrm{C}$ in a humidified $5 \% \mathrm{CO}_{2}$ atmosphere. The apoptosis was induced by actinomycin D (Sigma-Aldrich) treatment. The cells, centrifuged at $1200 \mathrm{rpm}$ for 10 min, were suspended in RPMI medium containing $50 \mu \mathrm{M}$ actinomycin D. For FTIR measurements $10^{7}$ cells $/ \mathrm{ml}$ were centrifuged, washed twice in PBS and resuspended in PBS; the spectra were collected at 2, 3.5, 7 hours after treatment.

Apoptosis was assayed using flow cytometry. Apoptotic cells percentage was determined by using Bender Med Systems Annexin V-FTIC apoptosis kit. $1 \times 10^{5}-1 \times 10^{6}$ cells were rinsed with HEPES buffer and resuspended in $200 \mu \mathrm{l}$ of the HEPES buffer. Then $5 \mu \mathrm{l}$ of annexin $\mathrm{V}$ and $10 \mu \mathrm{l}$ of propidium iodide were added to the suspension. Cells incubated at room temperature for 5-15 $\mathrm{min}$ in the dark were then analysed at 2, 3.5, 7 hours after treatment by flow cytometry (FACSCalibur BD Biosciences, San Jose, California, USA) at $488 \mathrm{~nm}$.

Cellular necrosis was induced lowering the $\mathrm{pH}$ value to 4.2 by adding $0.4 \mathrm{ml}$ of $\mathrm{HCl} 1 \mathrm{M}$ to cell culture. Viability was determined by the trypan blue exclusion test: $92 \%$ of necrotic cells were detected after 28 hours. 


\subsection{FTIR spectroscopy}

Spectra were recorded, by using the Attenuated Total Reflectance (ATR) mode, in the $3000-900 \mathrm{~cm}^{-1}$ region on a FT-IR/410 Jasco spectrometer equipped with a conductive ceramic coil mounted in a water cooled copper jacket source, a KBr beamsplitter and a TGS detector. The substrate used was a $\mathrm{ZnSe}$ crystal: its refractive index of 2.4 and an incident angle of $45^{\circ}$ obtained a total of six internal reflections at the sample.

Before the FT-IR measurements the cells, washed 2 times with PBS, centrifuged, resuspended in PBS, were transferred to the ZnSe optical plate. The recording conditions for each FT-IR spectrum were: 180 scans, a triangular apodization function and a resolution of $4 \mathrm{~cm}^{-1}$.

The preliminary reduction of IR spectral data (subtraction, normalization, etc.) was performed using the Spectra Manager Analysis software provided by Jasco. The spectral measurements were performed simultaneously with the apoptosis assay at 2, 3.5, 7 hours after the actinomycin D treatment.

\section{Results and discussion}

\subsection{Flow cytometry}

The flow cytometry results are reported in Fig. 1 (panels a, b, c, d) and summarized in Table 1.
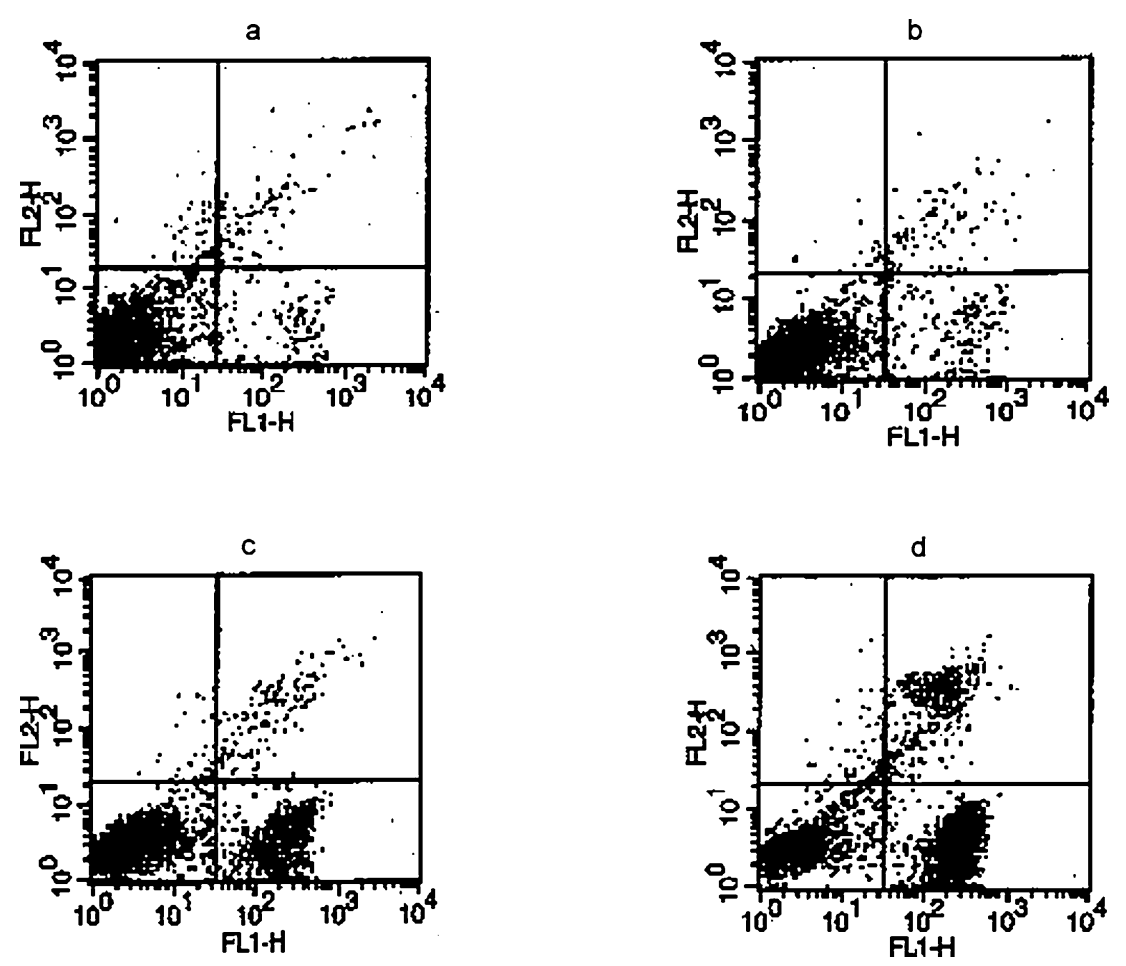

Fig. 1. Flow cytometry pictures concerning Jurkat cells: untreated (panel a); 2 hours after treatment with actinomycin D (panel b); 3.5 hours after treatment with actinomycin D (panel c); 7 hours after treatment with actinomycin D (panel d). The four quadrants in each panel correspond, respectively, to: necrotic cells (upper left), apoptotic late cells (upper right), apoptotic early cells (low right); viable cells (low left). 
Table 1

Flow cytometry results

\begin{tabular}{lcccc}
\hline Sample & \% viable & \% apoptotic early & \% apoptotic late & \% necrotic \\
\hline Control & 92.76 & 3.69 & 2.34 & 1.21 \\
AD2 & 91.03 & 5.57 & 2.79 & 0.61 \\
AD3.5 & 67.01 & 27.64 & 4.15 & 1.20 \\
AD7 & 36.77 & 47.90 & 13.51 & 1.82 \\
\hline
\end{tabular}

$\mathrm{AD} 2, \mathrm{AD} 3.5, \mathrm{AD} 7$ are the actinomycin $\mathrm{D}$ treated samples after 2 hours, 3.5 hours, 7 hours, respectively.

Cytometric measurements were performed at the same time of the spectroscopic ones by utilizing cells belonging to the same initial sample.

The four quadrants in each panel of Fig. 1 correspond, respectively, to: necrotic cells (upper left), apoptotic late cells (upper right), apoptotic early cells (low right), viable cell (low left).

FL1 on the $\mathrm{x}$-axis indicates the fluorescence intensity due to annexin V; FL2 on the y-axis indicates the fluorescence intensity of propidium iodide.

Table 1 reports the percentage values deduced from Fig. 1 of viable, necrotic and apoptotic cells $2 \mathrm{~h}$, $3.5 \mathrm{~h}, 7 \mathrm{~h}$ after actinomycin D treatment, respectively.

The data reported in Table 1 suggest that: (i) after each treatment, the percentage of necrotic cells is about the same and always lower than $2 \%$; this allows us to interpret the IR spectral features as characterizing the apoptotic processes; (ii) the apoptotic cells percentage increases as function of time till to $61 \%$.

\subsection{FT-IR spectroscopy}

All FT-IR obtained spectra exhibited a good signal-to-noise ratio and were highly reproducible.

Figure 2 shows the spectrum (absorbance in arbitrary units versus wavenumber in $\mathrm{cm}^{-1}$ ) of the control sample utilized for the analysis and the interpretation of the apoptotic and necrotic spectra.

As already partially described in our recent paper [2] and references therein, the different absorption bands due to the high number of vibrational modes interacting with the physical and chemical environment can be fitted by the addition of the major macromolecules contributions as proteins, lipids, carbohydrates and polynucleotides and can be summarized as follows:

(1) $\mathrm{CH}_{3}$ antisymmetric stretch at $2960 \mathrm{~cm}^{-1}$ due to methyl terminal group of the membrane phospholipids;

(2) $\mathrm{CH}_{2}$ antisymmetric stretch at $2928 \mathrm{~cm}^{-1}$ due to methylene group of the membrane phospholipids;

(3) $\mathrm{CH}_{2}$ symmetric stretch at $2856 \mathrm{~cm}^{-1}$ due to methylene group of the membrane phospholipids;

(4) amideI centered at $1640 \mathrm{~cm}^{-1}$, due to $\mathrm{C}=\mathrm{O}$ stretching vibration of the amide $\mathrm{C}=\mathrm{O}$ coupled to $\mathrm{NH}_{2}$ in planebending vibrations;

(5) the amideII absorption feature at $1550 \mathrm{~cm}^{-1}$ due to $\mathrm{N}-\mathrm{H}$ bending coupled to a $\mathrm{C}-\mathrm{N}$ stretching of the cell proteins;

(6) the $\mathrm{CH}_{2}$ deformation modes of lipids methylene group at $1455 \mathrm{~cm}^{-1}$;

(7) a band at $1397 \mathrm{~cm}^{-1}$ from $\mathrm{CH}_{3}$ bending vibration due to methyl bond in the membrane;

(8) the antisymmetric phosphate stretching arising from nucleic acid and DNA at $1235 \mathrm{~cm}^{-1}$; 


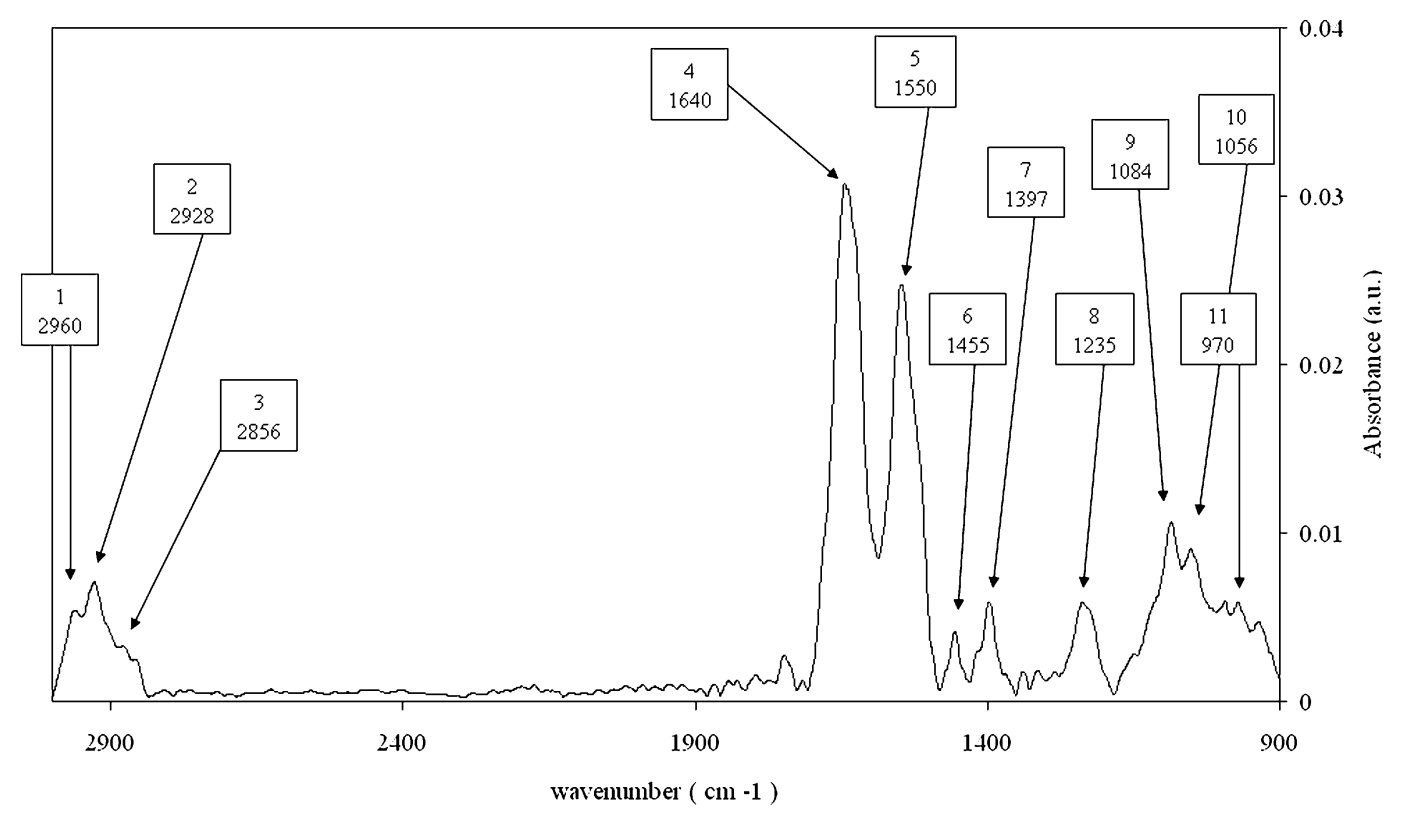

Fig. 2. IR spectrum of the control sample; the main features are labelled.

(9) the absorption band at $1084 \mathrm{~cm}^{-1}$ due to the different following components: $\mathrm{C}-\mathrm{O}$ stretching vibrations from nucleic acid sugar, the symmetric phosphate stretch and the $\mathrm{C}-\mathrm{O}-\mathrm{P}$ stretching vibration from phosphorylated lipids;

(10) the RNA nucleic acid ribose $\mathrm{C}-\mathrm{O}$ vibration band at $1056 \mathrm{~cm}^{-1}$;

(11) the DNA skeleton $\mathrm{C}-\mathrm{C} \backslash \mathrm{C}-\mathrm{O}$ stretching vibration at $970 \mathrm{~cm}^{-1}$.

The spectra obtained from the apoptotic and necrotic cells reveal a high number of significant changes to be accurately investigated. At first, we decided to concentrate our attention on the spectral region between 3000 and $2800 \mathrm{~cm}^{-1}$ where an immediate correlation with the data from the flow cytometry measurements seems to be evident. It is known that measurements of $\mathrm{CH}_{2}$ stretching, scissoring and rocking band are used to probe the physical state of lipid under various conditions [8].

In Fig. 3 this selected spectral region is reported; the five different profiles correspond (from bottom to upper) to following samples: necrotic, control, actinomycin D $2 \mathrm{~h}$ (2 hours after treatment), actinomycin D $3.5 \mathrm{~h}$, actinomycin D $7 \mathrm{~h}$.

By comparing the five profiles between each other we can see that (a) the absorption profile of the necrotic cells is very different with respect both to the control one and to the apoptotic spectra evolving with the time; in particular, the absorption bands corresponding to the methyl and methylene groups of the membrane's phospholipids disappear; (b) the three spectral bands centred at 2960, 2928 and $2856 \mathrm{~cm}^{-1}$ are more and more evident with the time in the apoptotic spectra, due to the progressive increase of their absorption intensity.

For each spectrum the total area of the $3000-2800 \mathrm{~cm}^{-1}$ spectral region has been evaluated.

The histograms in Fig. 4 show the behaviour of the areas of the apoptotic cells spectra versus the time compared with the area of both control and necrotic cells spectra.

Being the value of the necrotic cells spectrum area lower than $50 \%$ of the control cell spectrum area, it is evident that a high number of lipids molecules is no more present in the cells revealing a damage of 


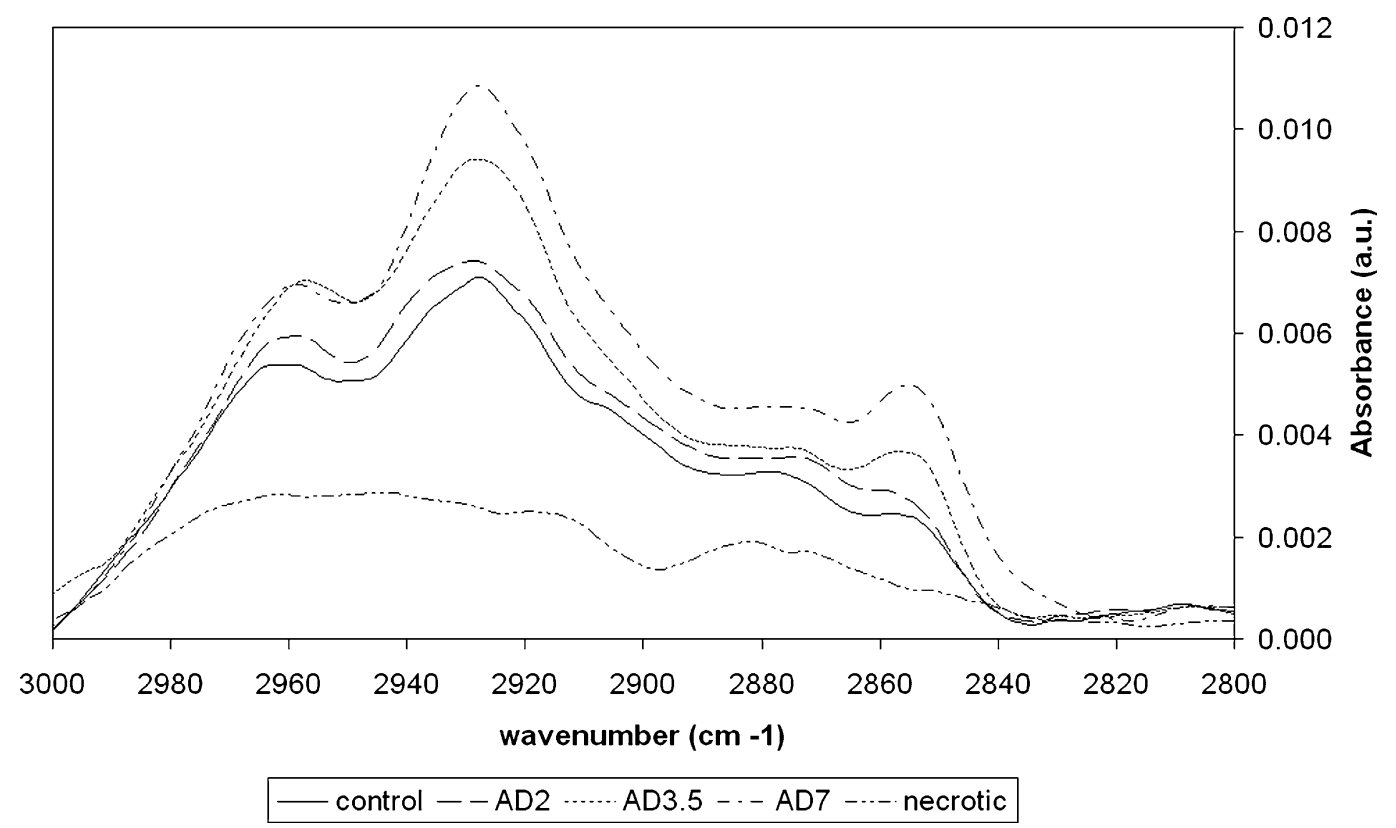

Fig. 3. 3000-2800 cm $\mathrm{cm}^{-1}$ IR spectra of Jurkat cells: (from bottom to upper) necrotic, untreated, 2 hours after treatment with actinomycin D (AD2), 3.5 hours after treatment with actinomycin D (AD3.5), 7 hours after treatment with actinomycin D (AD7).

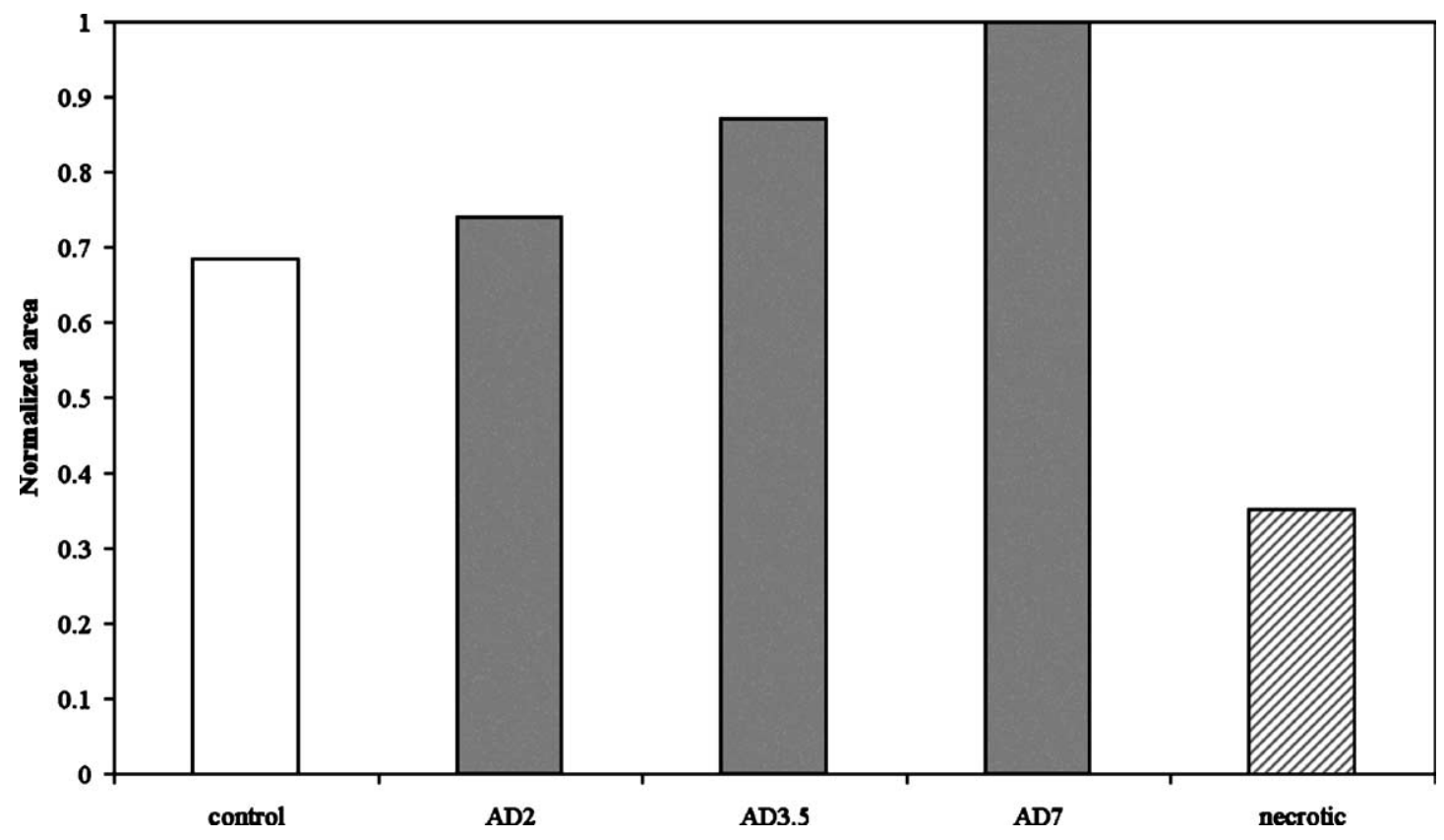

Fig. 4. The areas of the apoptotic cells spectra in $3000-2800 \mathrm{~cm}^{-1}$ range at different times after treatment, compared with the areas of both control and necrotic cells spectra. 


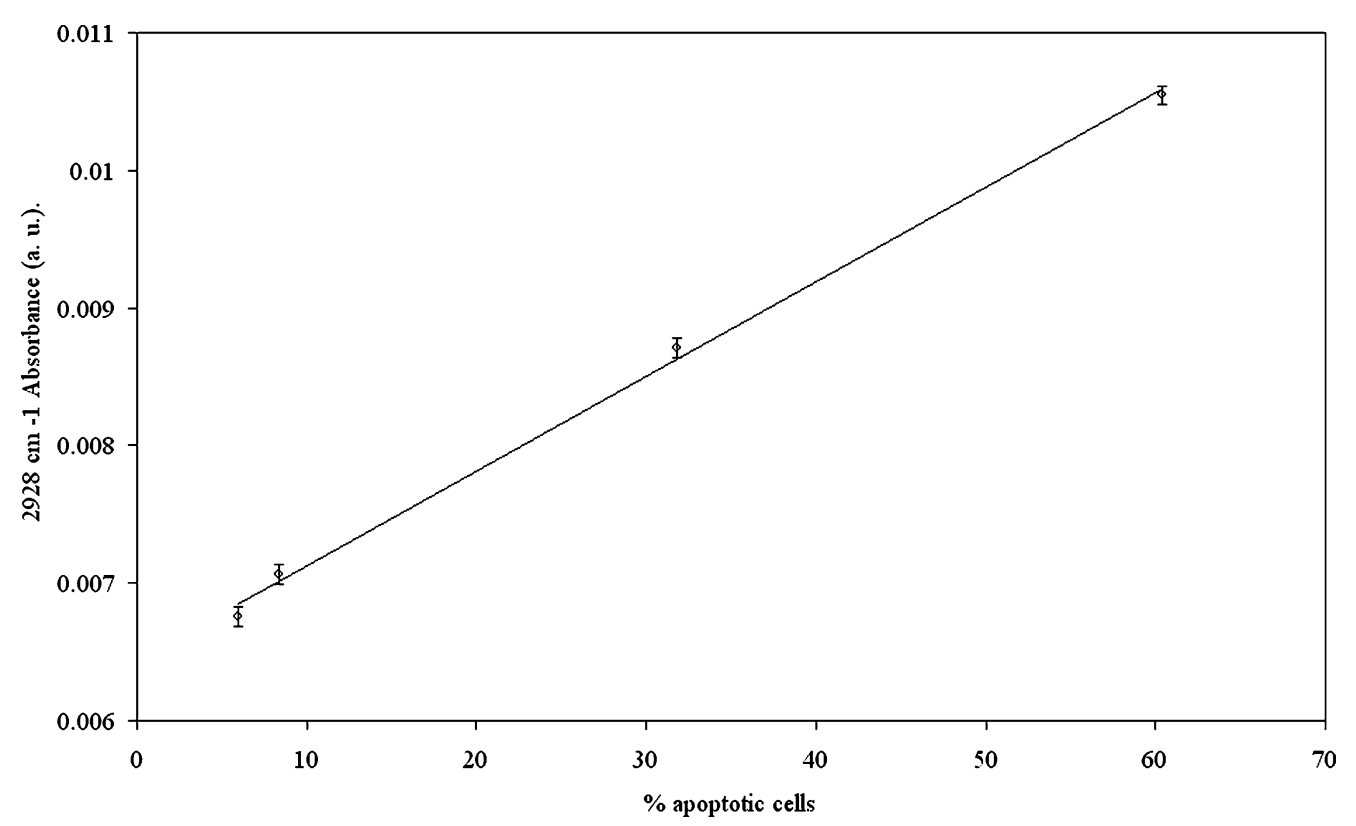

Fig. 5. The absorbance maximum value of the spectral band centred at $2928 \mathrm{~cm}^{-1}$ versus the $\%$ values of apoptotic cells calculated from flow cytometry measurements.

the membrane; on the other hand, the absence in the spectral profile of some definite bands and peaks suggests an advanced damage state.

The increase of the area is due to increase both of bandwidths and of the absorption intensity: while a bandwidths increasing due to the lipid $\mathrm{CH}_{2}$ vibrational modes indicates an increase of conformational disorder, the increasing of intensity is directly related to the number of molecular groups vibrating at the same frequencies.

In Fig. 5 the maximum value of the absorbance centred at $2928 \mathrm{~cm}^{-1}$ (assigned to $\mathrm{CH}_{2}$ antisymmetric stretch of the methylene group) versus the percentage values of the apoptotic cells obtained by flow cytometry measurements is reported.

Even for the other two bands, respectively centred at 2960 and $2856 \mathrm{~cm}^{-1}$, an increase of the maximum value's intensity with the increase of the apoptotic cells percentage is observed, but a strong linear correlation is revealed for the $2928 \mathrm{~cm}^{-1}$ band. The computed linear correlation factor is $r=0.999$ corresponding to a correlation probability of $99.9 \%$; the calculated angular coefficient of $(8.3 \pm 0.4) \times 10^{-5}$ a.u. shows a direct proportionality between the absorption intensity and the apoptotic cell's percentage.

\section{Conclusions}

The comparison between the results obtained on Jurkat cells, by using combined measurements series of FTIR spectroscopy and flow cytometry, confirms that the apoptosis induces a conformational disorder of lipid acyl chains, as elsewhere reported [8], and allows us to identify a spectral marker of apoptosis process corresponding to the phosphatidylserine externalization. This molecule, usually confined to the interior of the cell, exposes its polar head to the outer leaflet of the plasma membrane during the apoptosis process. 
The increase of the $2928 \mathrm{~cm}^{-1}$ absorption peak intensity during the apoptotic processes together with its linear correlation with the flow cytometry data, reflects changes in the amount and/or type of molecular groups vibrating at the same frequency.

As above described, the flow cytometry detects cells stained with annexin V bounded to the polar head of phosphatidylserine exposed to the outer leaflet of membrane; on the other hand, due to scramblase activation and the loss of lipid asymmetry [9], during the apoptosis process, the phosphatidylserine changes its orientation assuming the same orientation of other membrane phospholipids. Therefore the contribution of $\mathrm{CH}_{2}$ groups of phosphatidylserine to the absorption spectrum, by adding to that of other equally oriented phospholipids, increases the absorption intensity values.

Thus this band can be considered as a highly specific spectroscopic marker of apoptotic processes induced in Jurkat cells by actinomycin D, by signalling the externalization of phosphatidylserine.

In conclusion our results confirm that FTIR spectroscopy can be used as a powerful tool to detect cellular processes like apoptosis and necrosis. We plan to perform other experiments utilizing different agents to induce cellular apoptosis.

\section{Acknowledgement}

Many thanks to Dr. Stefano Belardinelli for his qualified assistance in the laboratory experiments.

\section{References}

[1] S. Arur, U.E. Uche, K. Rezaul, M. Fong, V. Scanton, A.E. Cowa, W. Mohler and d D.K. Han, Annexin I is an endogenous ligand that mediates apoptotic cell engulfment, Development Cell 4 (2003), 587-598.

[2] S. Gaudenzi, M.G. Furfaro, D. Pozzi, I. Silvestri and A. Congiu Castellano, Cell-metal interaction studied by cytotoxic and FT-IR spectroscopic methods, Environm. Toxic. Pharmac. 14 (2003), 51-59.

[3] N. Jamin, L. Miller, J. Moncuit, W.H. Fridman, P. Dumas and J.L. Teillaud, Chemical heterogeneity in cell death: combined synchrotron IR and fluorescence microscopy studies of single apoptotic and necrotic cells, Biopolymers 72 (2003), 366-372.

[4] J.F.R. Kerr and B.V. Harmon, Definition and incidence of apoptosis: an historical perspective, in: Apoptosis: the Molecular Basis of Cell Death, L.D. Tomei and F.O. Cope, eds, Harbor Lab. Press, N.Y. Cold Spring, 1991, pp. 5-29.

[5] T. Levade, Y. Hannun and S. Spiegel, Lipids and apoptosis, Biochim. Biophys. Acta 1585 (2002), 51-225 and references therein.

[6] J.R. Mourant, Y.R. Yamada, S. Carpenter, L.R. Dominique and J.P. Freyer, FTIR spectroscopy demonstrates biochemical differences in mammalian cell cultures at different growth stages, Biophys. J. 85 (2003), 1938-1947.

[7] D. Naumann, FT-IR and FT-NIR Raman spectroscopy in biomedical research, Appl. Spectrosc. Rev. 36 (2001), 239-298 and references therein.

[8] L.K. Tamm and S.A. Tatulian, Infrared spectroscopy of proteins and peptides in lipid bilayers, Quart. Rev. Biophys. 30 (1997), 365-429 and references therein.

[9] P. Williamson and R.A. Schlegel, Transbilayer phospholipids movement and the clearance of apoptotic cells, Biochim. Biophys. Acta 1585 (2002), 53-63.

[10] U.T. Wirthmueller, T. Kurosaki, M.S. Marakami and J.V. Ravetch, Signal transduction by Fc $\gamma$ RIII (CD16) is mediated through the $\gamma$ chain, J. Exp. Med. 175 (1992), 1381-1384.

[11] J. Zhou, Z. Wang, S. Sun, M. Liu and H. Zhang, A rapid method for detecting conformational changes during differentiation and apoptosis of HL60 cells by Fourier transform infrared spectroscopy, Biotechn. Appl. Biochem. 33 (2001), $127-132$ and references therein.

[12] M. Zornig, A.O. Hueber, W. Baum and G. Evan, Apoptosis regulators and their role in tumorigenesis, Biochim. Biophys. Acta 1551 (2001), F1-F37. 


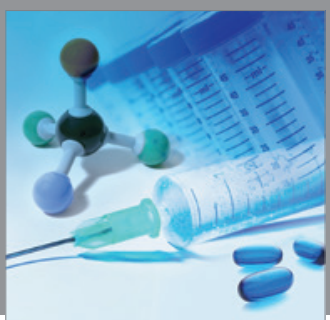

International Journal of

Medicinal Chemistry

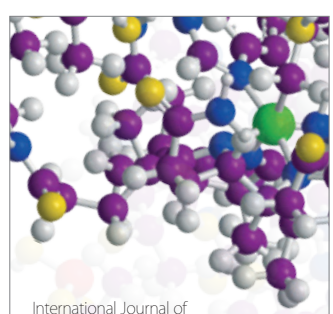

Carbohydrate Chemistry

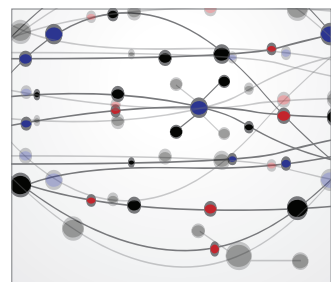

The Scientific World Journal
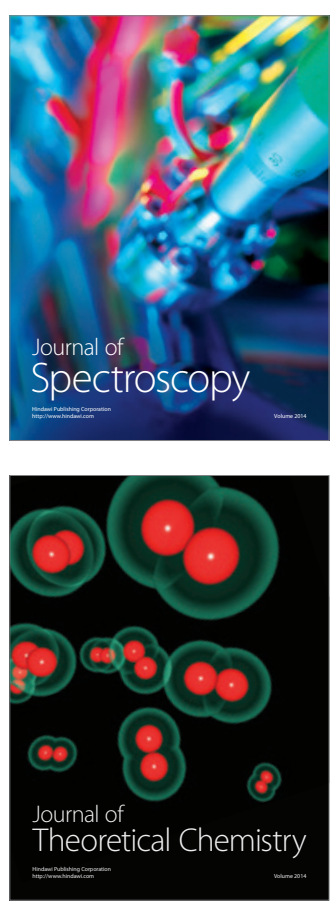
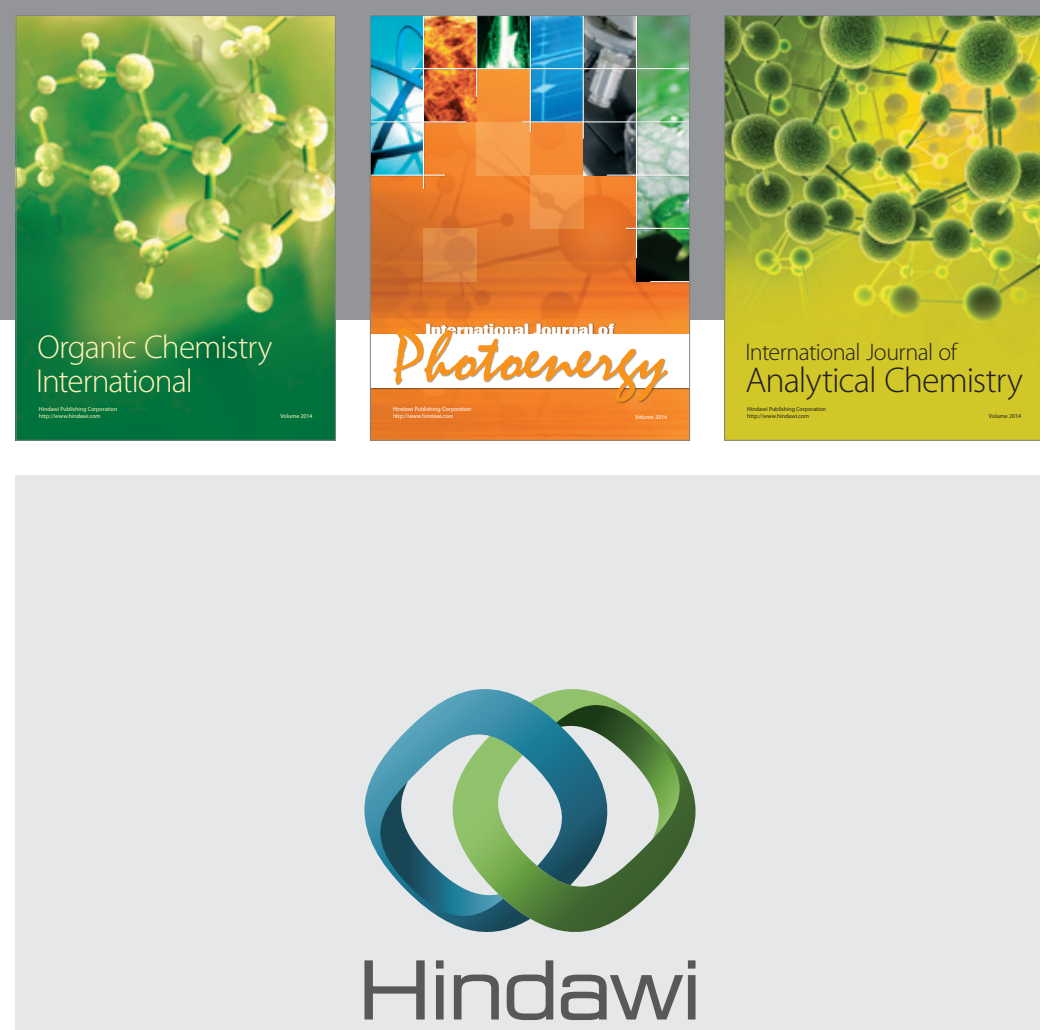

Submit your manuscripts at

http://www.hindawi.com
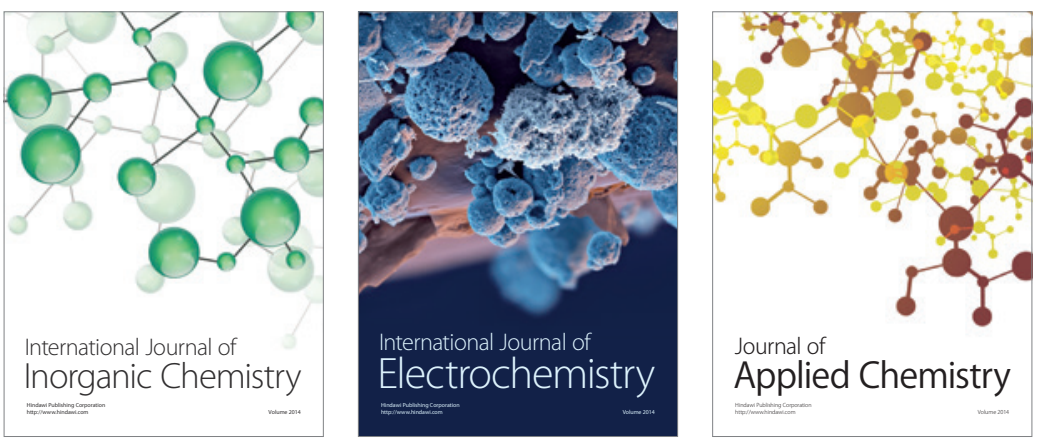

Journal of

Applied Chemistry
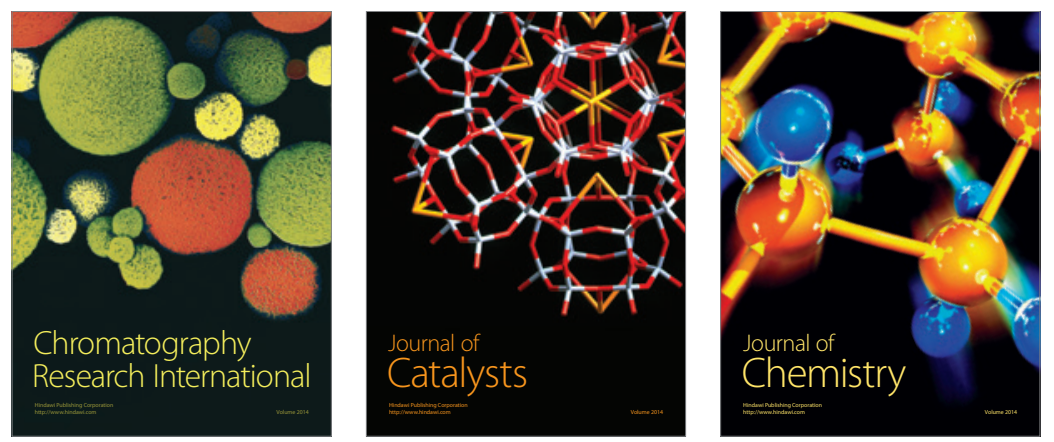
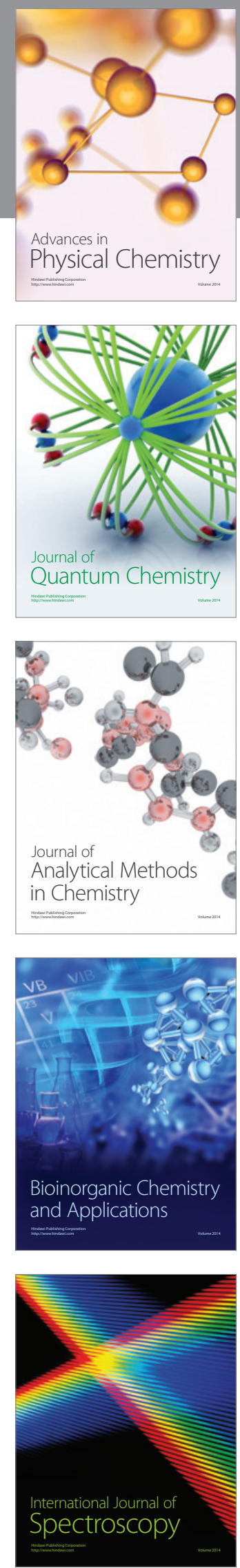